\title{
The efficacy and safety of irsogladine maleate in nonsteroidal anti-inflammatory drug or aspirin-induced peptic ulcer and gastritis
}

Ki-Nam Shim ${ }^{1,}$, Jin Il Kim ${ }^{2,}$, Nayoung Kim³, ${ }^{3,4}$ Sang Gyun Kim ${ }^{4}$, Yun Ju Jo ${ }^{5}$, Su Jin Hong ${ }^{6}$, Jeong Eun Shin ${ }^{7}$, Gwang Ha Kim ${ }^{8}$, Kyung Sik Park ${ }^{9}$, Suck Chei Choi ${ }^{10}$, Joong Goo Kwon ${ }^{11}$, Jie-Hyun $\mathrm{Kim}^{12}$, Hyun Jin $\mathrm{Kim}^{13}$, and Ji Won Kim ${ }^{14}$

${ }^{1}$ Department of Internal Medicine, College of Medicine, Ewha Womans University, Ewha Medical Research Institute, Seoul; ${ }^{2}$ Department of Internal Medicine, College of Medicine, The Catholic University of Korea, Seoul; ${ }^{3}$ Department of Internal Medicine, Seoul National University Bundang Hospital, Seongnam; ${ }^{4}$ Department of Internal Medicine and Liver Research Institute, Seoul National University College of Medicine, Seoul; ${ }^{5}$ Department of Internal Medicine, Eulji University School of Medicine, Seoul; ${ }^{6}$ Digestive Disease Center and Research Institute, Department of Internal Medicine, Soonchunhyang University College of Medicine, Bucheon; ${ }^{7}$ Department of Internal Medicine, Dankook University College of Medicine, Cheonan; ${ }^{8}$ Department of Internal Medicine, Pusan National University School of Medicine, and Biomedical Research Institute, Pusan National University Hospital, Busan; ${ }^{9}$ Department of Internal Medicine, Keimyung University School of Medicine, Daegu; ${ }^{10}$ Department of Internal Medicine, Wonkwang University College of Medicine and Digestive Disease Research Institute, Iksan; ${ }^{11}$ Department of Internal Medicine, Daegu Catholic University School of Medicine, Daegu; ${ }^{12}$ Department of Internal Medicine, Gangnam Severance Hospital, Yonsei University College of Medicine, Seoul; ${ }^{13}$ Department of Internal Medicine, Gyeongsang National University School of Medicine, Jinju; ${ }^{14}$ Department of Internal Medicine, SMG-SNU Boramae Medical Center, Seoul, Korea

Background/Aims: Irsogladine maleate, an enhancer of gastric mucosal protective factors, has demonstrated its efficacy for various gastric mucosal injuries. The aim of this study was to evaluate the efficacy and safety of irsogladine for prevention of nonsteroidal anti-inflammatory drugs (NSAIDs) or aspirin-induced peptic ulcer and gastritis.

Methods: In this multicenter, randomized, double-blind, exploratory clinical trial, 100 patients over 50 years of age who needed continuous NSAIDs or aspirin for more than 8 weeks were randomly assigned to either test group (irsogladine maleate $2 \mathrm{mg}$, twice daily, 39 patients for full analysis) or placebo group (37 patients for full analysis). Primary outcomes were incidence of peptic ulcer and ratio of modified Lanza score (MLS) 2 to 4 . Secondary outcome was the number of acute erosions confirmed by endoscopy at 8 weeks. Adverse effects were also compared.

Results: There were no significant differences in gastric protective effects between test and placebo groups. However, two cases of peptic ulcer in the placebo group but none in the test group were observed. These two cases of peptic ulcer were Helicobacter pylori-negative. In addition, $H$. pylori-negative group showed significant changes in MLS score $(p=0.0247)$ and edema score $(p=0.0154)$ after the treatment compared to those before treatment in the test group. There was no significant difference in adverse events between the two groups.

Conclusions: The efficacy of irsogladine maleate was found in $\mathrm{H}$. pylori-negative group, suggesting its potential as a protective agent against NSAIDs or aspirin-induced peptic ulcer and gastritis.

Keywords: Irsogladine maleate; Anti-inflammatory agents, non-steroidal; Aspirin; Peptic ulcer; Gastritis

Received: November 5, 2017

Revised : January 28, 2018 Accepted: February 21, 2018

\section{Correspondence to}

Nayoung Kim, M.D.

Department of Internal Medicine, Seoul National University Bundang Hospital, 82 Gumi-ro 173beon-gil, Bundang-gu, Seongnam 13620, Korea

Tel: +82-31-787-7008, Fax: +82-31-787-4051

E-mail: nayoungkim49@empas.com

${ }^{\star}$ These authors contributed equally to this work. 


\section{INTRODUCTION}

Nonsteroidal anti-inflammatory drugs (NSAIDs) are widely used for treating various types of acute and chronic musculoskeletal disorders. According to the Korean National Statistical Office's data on population distribution by age group, the elderly people aged 65 and over accounted for $13.8 \%$ of the total population in 2017 . It is expected to increase to $24.3 \%$ in 2030 and $37.4 \%$ in 2050. When the population is getting older, the number of patients with chronic diseases such as musculoskeletal diseases and cerebrovascular diseases will increase. Therefore, the use of NSAIDs or aspirin is expected to continue to increase. The widespread use of NSAIDs raises the risk of drug side effects, including gastrointestinal (GI) damage. The risk of gastric mucosal defects (erosion or petechiae) in patients with long-term use of NSAIDs has been reported to be about 50\%. The rate of ulcers is about $1 \%$ and the rate of ulcers with complications is reported to be up to $0.5 \%$ per year $[1,2]$. However, in patients who have previous history of peptic ulcers with old age of more than 65 years old and concurrent use with other NSAIDs or high dose NSAIDs, steroids, and other anticoagulants [3], peptic ulcer can occur frequently [4]. High frequency of peptic ulcer and its complications in aged people $[5,6]$ is caused by decreased gastric mucosal protection provided by connective tissue due to accumulation of oxidative products when people are getting old [7]. In addition, impairment of apoptosis, angiogenesis, and sensory neuron activity via activation of early growth response-1 (Egr-1), phosphatase and tension homologue deleted on chromosome 10 (PTEN) might increase the susceptibility of gastric mucosa to injury during aging [7]. Thus, misoprostol and proton pump inhibitor (PPI) preparations are usually recommended to prevent NSAID or aspirin-induced peptic ulcer and gastritis [8], especially for the elderly. Infection by Helicobacter pylori is more likely to cause peptic ulcers if patients take NSAIDs. Therefore, those who take longterm NSAIDs are recommended to treat $H$. pylori eradication [9]. However, $H$. pylori eradication alone cannot reduce the incidence of ulcers [10]. In addition, aspirin is widely used in diseases such as cardiovascular thrombosis and rheumatoid arthritis. When taken daily at low doses, aspirin may damage the mucous membrane of the stomach and cause ulcers or bleeding [11-15]. Treat- ment guidelines of NSAIDs-induced GI injury are based on prevention of peptic ulcer and gastritis. The most effective method of primary prevention for peptic ulcer caused by NSAIDs is to stop using the drug or not using the drug. However, most patients are unable to discontinue the medication. Therefore, it is important to find strategic preventive measures through risk assessment [16]. In order to prevent the development of peptic ulcer caused by NSAIDs or aspirin, histamine 2 receptor antagonist ( $\mathrm{H}_{2} \mathrm{RA}$ ) alone is less effective compared to simultaneous administration of misoprostol or PPI. Misoprostol is effective for preventing GI complications caused by NSAIDs. However, it can cause GI complications such as diarrhea accompanied by convulsive abdominal pain. In addition, it has recently been reported that long term use of PPI can cause various side effects [17], and there are limitations in terms of insurance coverage for long-term prophylactic use. Therefore, developing gastroprotective drugs useful for preventing GI complications due to long-term use of NSAIDs or aspirin is essential.

Irsogladine maleate (Gaslon $\mathrm{N}^{\circledR} \mathrm{OD}$, Taejoon Pharm Co. Ltd., Seoul, Korea) is an enhancer of gastric mucosal protective factors. It increases the production of intracellular cyclic adenosine monophosphate (cAMP) by inhibiting phosphodiesterase activity [18]. It can activate intercellular communication [19], prevent reduction in gastric mucosal blood flow [20,21], increase anti-inflammatory activity [18], and prevent reduction in mucosal hydrophobicity [22]. Although its usefulness has been demonstrated in various models of gastric mucosal injury, its efficacy of protection for NSAIDs or aspirin-induced GI damage, especially in people with old age, is currently unknown. Therefore, the objective of this study was to evaluate the efficacy and safety of irsogladine maleate against peptic ulcer and gastritis caused by long-term use of NSAIDs or aspirin in people who were over 50 years old.

\section{METHODS}

\section{Study participants}

Eligible patients were more than 50 years old who were taking NSAIDs (including cyclooxygenase 2 [COX-2] inhibitor, multiple NSAIDs) or aspirin (more than 80 
mg/day) for more than 8 weeks. They had baseline endoscopic findings without active peptic ulcer or gastric erosions. Written informed consent was obtained from each patient before participating in this study.

Exclusion criteria were history of gastric acid inhibiting or esophagogastric surgery, Zollinger-Ellison syndrome, active peptic ulcer or active erosive gastritis at screening endoscopy, reflux esophagitis more than LA-A, bleeding tendency or coagulopathy, inflammatory bowel disease, malabsorption and esophageal stricture within 3 months, hypersensitivity to irsogladine maleate, any use of PPI, H2RA, muscarinic receptor antagonist, corticosteroid, antacid, mucoprotective agents, anticoagulants (except aspirin), anti-thrombotic agents, bisphosphonate, anti-convulsant, anti-cholinergics, and promotility drug within 2 weeks, genetic problems such as galactose intolerance, lactase deficiency, and glucose-galactose malabsorption, any malignancy within 5 years, pregnant or lactating females, and females of childbearing age not using contraception.

\section{Study design}

This study was a multicenter, randomized, double-blind, exploratory clinical trial conducted in South Korea from December 2014 to September 2016. Patients meeting the study criteria underwent screening endoscopy, laboratory tests, and urea breath test (UBT) for H. pylori. Eligible patients were randomly assigned to either irsogladine maleate (Gaslon $\mathrm{N}^{\circledast} \mathrm{OD}, 2 \mathrm{mg}$ twice a day) or placebo treatment group. Irsogladine maleate and placebo were taken twice daily for 8 weeks, concurrent with NSAIDs or aspirin.

An independent statistical office (Seoul CRO Co. Ltd., Seoul, Korea) performed permuted stratified block randomization using block size 2 or 4 to stratify study site. They used SAS version 9.2 (SAS institute Inc., Cary, NC, USA) for sequence generation to stratify participating center. Random sequence was sent to each center via an interactive web-based response system to ensure allocation concealment. Patients with a final compliance of less than $80 \%$ were excluded from per protocol (PP) analysis. Among 100 eligible patients, 88 subjects were randomly assigned to this study. Of these 88 subjects, 87 (98.86\%) were received the clinical trial drug. However, only 76 (86.36\%) completed the clinical trial. Reasons for withdrawal included withdrawal of consent $(n=8$, $9.09 \%)$, and decision of the investigator $(n=3,3.41 \%)$. Of
88 randomized patients (100\%), 87 (98.86\%) were included in the safety arm, of which 76 (86.36\%) were included in the intention-to-treat (ITT) group while 11 patients (12.50\%) were excluded due to misuse of medication and misuse of IP. A total of 65 patients (73.86\%) were admitted to PP group. Fig. 1 presents a flowchart of patient progression through the study with reasons for premature discontinuation. The subject visited each center for follow-up at week 4 and week 8 .

\section{Number of target subjects and basis of calculation}

In order to see the expected effect between the test group and the control group, the incidence of peptic ulcer should be predicted based on previous studies. However, since there is no previous study on irsogladine maleate, we used previous studies regarding difference in the incidence of peptic ulcer expected to be statistically tested [23-30]. In this study, we referred to other studies conducted on patients with peptic ulcer due to prolonged administration of NSAIDs. The incidence of peptic ulcers was $21.7 \%$ in the placebo group and $1.4 \%$ in the group prescribed $200 \mu \mathrm{g}$ of misoprostol for 12 weeks for prevention and treatment of stomach and duodenal ulcer as an analogue of prostaglandin E1 [24]. Based on this, it was possible to calculate that 44 subjects were required for each group using the following formula by setting the first kind error of $5 \%$, $80 \%$ statistical power, the test of both sides, and a dropout rate of $20 \%$.

$$
n=\frac{\left(z_{\alpha / 2}+z_{\beta}\right)^{2}\left(p_{c} q_{c}+p_{t} q_{t}\right)}{\left(p_{c}-p_{t}\right)^{2}}
$$

\section{Upper GI endoscopy and measurement of malondi- aldehyde level}

Endoscopy and malondialdehyde (MDA, a lipid peroxidation product) level measurements were performed at Visit 1 (day o) and Visit 3 (8 weeks \pm 20 days). Standard and near or far range photo takings were performed according to guidelines for upper GI endoscopy. Diagnosis and evaluation of endoscopic photographs were conducted by the investigator of each institution. Separately, endoscopic photographs were collected at the center using a portable storage device. Diagnosis and evaluation were carried out by two independent evaluators. 


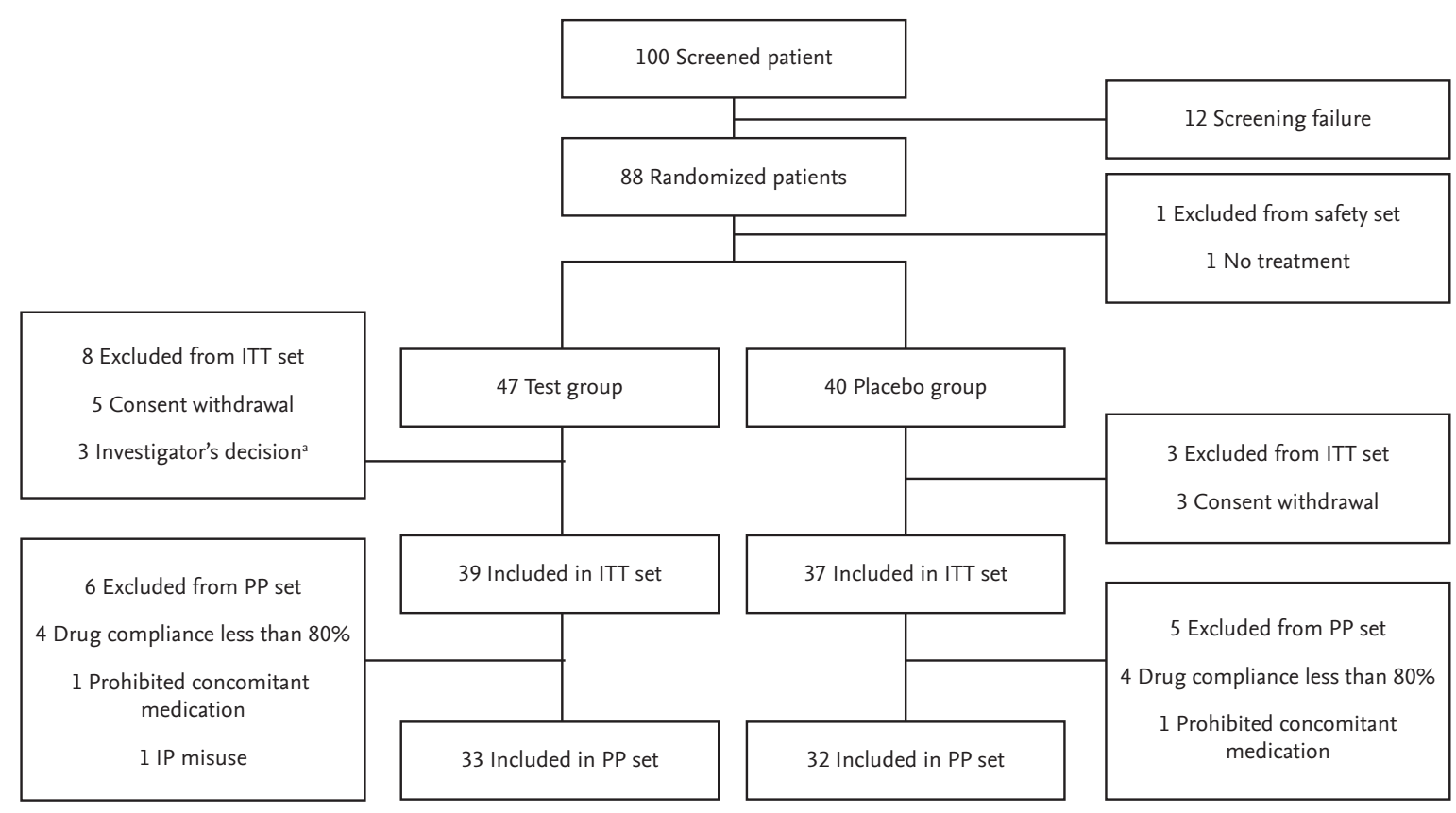

Figure 1. Flow chart showing enrolled patients and dropouts from the study. ITT, intention-to-treat; PP, per protocol; IP, investigational product. ${ }^{a}$ Adverse reaction not related to study drug: edema pheripheral $(n=1)$, dizziness and urticaria $(n=1)$, and chest discomfort $(n=1)$.

MDA was measured as a surrogate marker to assess the degree of oxidative stress and antioxidant effect of the test drug. However, MDA level measurement was omitted for subjects whose endoscopic examinations were waived because endoscopic examination of visit 1 (day o) was confirmed within 4 weeks of the internal examination and enough image results were confirmed to be suitable for participating in this study. MDA level was measured by biopsy in subjects who were confirmed to have ulcer by endoscopy. Cryotube for biopsy was labelled and provided to each institution. In principle, each institution should store the collected sample in a deep freezer for up to 2 months. These samples were then sent to the Central Lab (Seoul National University Bundang Hospital, Seongnam, Korea). Sent samples were used to measure level of MDA, a representative marker of peroxidation products known as the cause or result of GI tissue damage, by homogenization. Since there was no sample remaining after MDA measurement, the sample was automatically discarded. MDA was measured in tissue homogenate using ELISA kit (Sigma-Aldrich, St. Louis, MO, USA) [31]. MDA was extracted by homogenizing isolated gastric mucosa (10 $\mathrm{mg}$ ) in $300 \mu \mathrm{L}$ of MDA lysis buffer containing $3 \mu \mathrm{L}$ of $\mathrm{BHT}$ (100x) followed by centrifugation at 13,000 $\times g$ for
10 minutes. Thiobarbituric acid solution was added into each vial containing $200 \mu \mathrm{L}$ of standards and sample followed by incubation at $95^{\circ} \mathrm{C}$ for 60 minutes. After cooling to room temperature in an ice bath for 10 minutes, $200 \mu \mathrm{L}$ was taken from each mixture into a 96-well plate for analysis.

\section{Study assessments}

\section{Efficacy}

Each subject underwent an upper GI endoscopy to establish a baseline and again after the 8-week treatment period. Primary efficacy evaluation variables were incidence of peptic ulcer and gastric mucosal protection rate as a ratio of modified Lanza score (MLS) of 2 to 4 between test and control groups at 8 weeks (Table 1) $[32,33]$. Secondary efficacy evaluation variables were the number of acute erosions confirmed by endoscopy at 8 weeks, MDA level change, comparison of edema score, redness score, and hemorrhage score at 8 weeks between the test and control groups (Table 1) [34], and effective rate of subjective symptoms. The level of MDA was measured for two biopsy samples obtained from the greater curvature of mid antrum at initial and 8-week endoscopies. Two more biopsy samples were obtained at the 
Table 1. Endoscopic scoring of gastric mucosal lesion and mucosal injuries

\begin{tabular}{|c|c|}
\hline \multicolumn{2}{|c|}{ Gastric mucosal lesion } \\
\hline \multicolumn{2}{|c|}{ Modified Lanza score } \\
\hline o & No visible lesion \\
\hline 1 & Mucosal hemorrhages only \\
\hline 2 & One or two erosions \\
\hline 3 & Numerous (3-10) numbers of erosions \\
\hline 4 & Large (>10) numbers of erosions \\
\hline 5 & Ulcer \\
\hline \multicolumn{2}{|c|}{ Gastric mucosal injuries } \\
\hline \multicolumn{2}{|c|}{ Edema } \\
\hline 1 & No edema \\
\hline 2 & $\begin{array}{l}\text { Mucosa is somewhat pale, the white prom- } \\
\text { inence, and the hexagonal gastric pit be- } \\
\text { comes prominent }\end{array}$ \\
\hline \multicolumn{2}{|c|}{ Redness } \\
\hline 1 & No redness \\
\hline 2 & Mild reddish change \\
\hline 3 & More prominent reddish color change \\
\hline 4 & Beefy-reddish color change \\
\hline \multicolumn{2}{|c|}{ Hemorrhage } \\
\hline 1 & No hemorrhage \\
\hline 2 & Single hemorrhagic lesion \\
\hline 3 & 2-5 hemorrhagic lesions \\
\hline 4 & 6-10 hemorrhagic lesions \\
\hline 5 & $\begin{array}{l}\text { > } 10 \text { hemorrhagic lesions or larger area of a } \\
\text { confluent hemorrhage }\end{array}$ \\
\hline
\end{tabular}

ulcer edge in case of gastric ulcer occurrence for MDA level measurement.

\section{Safety}

Safety analysis was based on the safety analysis group and the number of adverse reactions and rescue drug use. In addition, the presence or absence of adverse events was compared using appropriate statistical analysis according to characteristics of variables.

\section{Statistical analysis}

Data obtained from subjects were analysed in three forms: safety group, ITT group, and PP group. Safety analysis included all data from randomly assigned subjects who took the study drug in the analysis. In the analysis of the ITT group, all subjects who had data on primary efficacy evaluation parameters after administration of the clinical trial drug were included in the analysis. The incidence of peptic ulcer by gastroscopy was analysed if missing data were obtained at a certain point in time. When the subject was dropped before the clinical trial was terminated, the latest data were obtained at that time (last observation carried forward method). Analysis of the PP group means analysis of the subjects included in the ITT analysis from the data obtained from the subjects completed according to the clinical trial plan. In principle, IT'T method was used as the main method for validity data. Additional PP analysis was performed when necessary. Safety data were principally based on safety analysis. Data on efficacy were used to perform subanalyses based on UBT test positive and negative results, and NSAIDs or aspirin naive and existing users.

Primary efficacy parameters were presented as frequency and ratio in each group. Chi-square test or Fisher's exact test was performed at significance level of $5 \%$ to test differences between groups. Secondary efficacy variables were descriptive statistics for continuous data. Paired $t$ test or Wilcoxon signed rank test was used to test differences between baseline and follow-up visits for both test and control groups. Independent $t$ test was performed to determine differences between groups. Frequency, proportion, and $95 \%$ confidence interval (CI) were presented for discrete data and chi-square test or Fisher exact test was performed to determine differences between groups. The percentage of subjects who developed adverse reactions and 95\% CI were presented for each group and chi-square test or Fisher's exact test was performed when difference between the test group and the control group was needed.

\section{Ethics statement}

This clinical trial was approved by the Institutional Review Board (IRB) of each participating institution ( $\mathrm{n}=$ 14) (IRB number of Seoul National University Bundang Hospital: B-1404/245-006).

\section{RESULTS}

\section{Baseline characteristics of study participants}

In the IT'T group, there were 35 males (46.05\%) and 41 fe- 
Table 2. Baseline characteristics of study participants

\begin{tabular}{|c|c|c|c|c|}
\hline Variable & Test group $(n=39)$ & Control group $(n=37)$ & $\operatorname{Total}(\mathrm{n}=76)$ & $p$ value $^{\mathrm{a}}$ \\
\hline \multicolumn{5}{|l|}{ Sex } \\
\hline Male & $18(46.15)$ & $17(45.95)$ & $35(46.05)$ & 0.9855 \\
\hline Female & $21(53.85)$ & $20(54.05)$ & $41(53.95)$ & \\
\hline \multicolumn{5}{|l|}{ Age, yr } \\
\hline Mean \pm SD & $65.33 \pm 7.55$ & $66.16 \pm 7.66$ & $65.74 \pm 7.57$ & 0.6363 \\
\hline Median & 66.00 & 68.00 & 66.00 & \\
\hline Min-Max & $53.00-81.00$ & $51.00-79.00$ & $51.00-81.00$ & \\
\hline \multicolumn{5}{|l|}{ Height, cm } \\
\hline Mean \pm SD & $160.57 \pm 6.94$ & $161.18 \pm 7.80$ & $160.87 \pm 7.33$ & 0.7210 \\
\hline Median & 160.00 & 162.00 & 160.80 & \\
\hline Min-Max & $148-175$ & $144-177$ & $144-177$ & \\
\hline \multicolumn{5}{|l|}{ Weight, kg } \\
\hline Mean \pm SD & $62.53 \pm 8.96$ & $64.17 \pm 10.13$ & $63.33 \pm 9.52$ & 0.4574 \\
\hline Median & 62.00 & 64.00 & 63.05 & \\
\hline Min-Max & $45.00-92.00$ & $46.50-86.00$ & $45.00-92.00$ & \\
\hline \multicolumn{5}{|l|}{ Smoking } \\
\hline Current smoking & $3(7.69)$ & $3(8.11)$ & $6(7.89)$ & 1.0000 \\
\hline Ex-smoking & $8(20.51)$ & $8(21.62)$ & $16(21.05)$ & \\
\hline Never & $28(71.79)$ & $26(70.27)$ & $54(71.05)$ & \\
\hline \multicolumn{5}{|l|}{ Helicobacter pylori infection } \\
\hline Positive & $13(33.33)$ & $15(40.54)$ & $28(36.84)$ & 0.5150 \\
\hline Negative & $26(66.67)$ & $22(59.46)$ & $48(63.16)$ & \\
\hline \multicolumn{5}{|l|}{ NSAIDs/aspirin use } \\
\hline Naïve & $11(28.21)$ & $14(37.84)$ & $25(32.89)$ & 0.3717 \\
\hline Existing user ${ }^{\mathrm{b}}$ & $28(71.79)$ & $23(62.16)$ & $51(67.11)$ & \\
\hline \multicolumn{5}{|l|}{ Current medical history } \\
\hline Musculoskeletal and connective tissue disorders & $10(25.64)$ & $7(18.92)$ & $17(22.37)$ & - \\
\hline Cardiac disorders & $11(28.21)$ & $7(18.92)$ & $18(23.68)$ & \\
\hline Nervous system disorders & $4(10.26)$ & $6(16.22)$ & $10(13.16)$ & \\
\hline Infections and infestations & $5(12.82)$ & $4(10.81)$ & $9(11.84)$ & \\
\hline Psychiatric disorders & $2(5.13)$ & $3(8.11)$ & $5(6.58)$ & \\
\hline Reproductive system and breast disorders & $6(15.38)$ & $1(2.70)$ & $7(9.21)$ & \\
\hline Respiratory, thoracic and mediastinal disorders & $2(5 \cdot 13)$ & $4(10.81)$ & $6(7.89)$ & \\
\hline Eye disorders & $3(7.69)$ & $\circ$ & $3(3.95)$ & \\
\hline Endocrine disorders & $3(7.69)$ & $2(5.41)$ & $5(6.58)$ & \\
\hline $\begin{array}{l}\text { General disorders and administration site condi- } \\
\text { tions }\end{array}$ & o & $4(10.81)$ & $4(5.26)$ & \\
\hline Injury, poisoning, and procedural complications & $3(7.69)$ & $1(2.70)$ & $4(5.26)$ & \\
\hline $\begin{array}{l}\text { Neoplasms benign, malignant and unspecified } \\
\text { (including cysts and polyps) }\end{array}$ & $3(7.69)$ & $1(2.70)$ & $4(5.26)$ & \\
\hline Congenital, familial, and genetic disorders & $2(5.13)$ & $1(2.70)$ & $3(3.95)$ & \\
\hline Hepatobiliary disorders & $1(2.56)$ & $2(5.41)$ & $3(3.95)$ & \\
\hline Blood and lymphatic system disorders & $1(2.56)$ & $1(2.70)$ & $2(2.63)$ & \\
\hline
\end{tabular}


Table 2. Continued

\begin{tabular}{lccc}
\hline Variable & Test group $(\mathrm{n}=39)$ & Control group $(\mathrm{n}=37)$ Total $(\mathrm{n}=76)^{p \text { value }}$ \\
\hline Skin and subcutaneous tissue disorders & $2(5.13)$ & 0 & $2(2.63)$ \\
Ear and labyrinth disorders & 0 & $1(2.70)$ & $1(1.32)$ \\
Investigations & $1(2.56)$ & 0 & $1(1.32)$ \\
Renal and urinary disorders & 0 & $1(2.70)$ & $1(1.32)$ \\
Social circumstances & 0 & $1(2.70)$ & $1(1.32)$ \\
Surgical and medical procedures & $1(2.56)$ & 0 & $1(1.32)$ \\
\hline
\end{tabular}

Values are presented as number (\%). Test group, irsogladine maleate (Gaslon $\left.\mathrm{N}^{\circledR} \mathrm{OD}\right), 2$ mg twice a day; Control group, placebo drug of irsogladine maleate $2 \mathrm{mg}$ twice a day.

SD, standard deviation; NSAID, nonsteroidal anti-inflammatory drug.

${ }^{a}$ Test group vs. control group (continuous variable, independent $t$ test; categorical variable, chi-square test; smoking, Fisher's exact test; $H$. pylori infection, NSAIDs/aspirin use, chi-square test).

${ }^{\mathrm{b}}$ Previous users were guided to participate after wash-out period longer than 7 days.

males (53.95\%). Their mean age, height, and body weight were $65.74 \pm 7.57$ years, $160.87 \pm 7.33 \mathrm{~cm}$, and $63.33 \pm 9.52 \mathrm{~kg}$, respectively. There was no statistically significant difference in any demographic characteristics including $H$. pylori infection status and history of NSAIDs or aspirin use between the two groups (Table 2). Medical history of subject was examined and presented as System Organ Class (SOC) and Preferred Term (PT) according to Medical Dictionary for Regulatory Activities (MedDRA) version 19.0 (MedDRA, McLean, VA, USA). There was no statistically significant difference in medical history between treatment groups, including 15 cases of past history of GI disorders in 13 patients (17.11\%) and 28 cases of past medical history in 18 patients $(23.68 \%)$ in the ITT group $(p=0.1358)$. Current history of continued use of NSAIDs or aspirin for more than 8 weeks was confirmed in 249 cases of 76 patients of ITT group (100\%), including cases of vascular disorders (49 cases in 49 patients, 64.47\%), metabolic and nutritional disorders (45 cases in 39 patients, $51.32 \%$ ), musculoskeletal and connective tissue disorders (22 cases in 17 patients, $22.37 \%$ ), and cardiac disorders (20 cases in 18 patients, $23.68 \%$ ), and nervous system disorders (12 cases in 10 patients, 13.16\%). Current status of GI disorders was found in 33 cases of 26 patients (34.21\%). Detailed medical history for the use of NSAIDs or aspirin was shown in Table 2.

\section{Efficacy}

Primary and secondary efficacy parameters in IT'T group and PP group were analysed. ITT group was divided into NSAIDs or aspirin naive and NSAIDs or aspirin partici- pating group with more than 7 days of wash-out period, $H$. pylori-positive group, and $H$. pylori-negative group.

\section{Primary efficacy assessment}

The ITT population had 76 patients (39 in the test group and 37 in the placebo group). Primary efficacy endpoint was MLS score of 5 and MLS score of 2 to 4 at 8 weeks. Results of endoscopic finding by institutional investigators were confirmed by two independent evaluators and consistency in MLS standard score was confirmed. Cohen's weighted $\kappa$ value for the agreement between institute investigator and independent evaluator 1 was 0.4707 (moderate concordance) and that between institute investigator and independent evaluator 2 was 0.3566 (fair concordance).

(1) Incidence of peptic ulcer: At the end of the 8th week after administering the test drug, the percentage of patients with peptic ulcer (MLS 5) was assessed. As a result, no subject (०\%) in the test group and two subjects (5.41\%) in the control group were found to have gastric ulcer (one case of peptic ulcer in aspirin-naive patient and one case in patient who was taking aspirin after abstinence for more than 7 days). Both cases occurred in $\mathrm{H}$. pylori-negative control patients. There was no statistical significance between groups (Table 3). Subgroup analysis did not show any statistical significance between groups either.

(2) Incidence of MLS: In endoscopic examination at 8 weeks after administering the test drug, 11 subjects $(28.21 \%)$ in the test group and eight patients $(21.62 \%)$ in the control group were found to have peptic inflammation with MLS of 2 to 4 . There was no statistically 
Table 3. Comparison of severity of mucosal injury based on modified Lanza score at 8 weeks after treatment

\begin{tabular}{|c|c|c|c|}
\hline Mucosa injury & Test group $(\mathrm{n}=39)$ & Control group $(\mathrm{n}=37)$ & $p$ value \\
\hline No occurrence (Grade $0-1$ ) & $28(71.79)$ & $27(72.97)$ & $0.4337^{\mathrm{a}}$ \\
\hline Occurrence (Grade 2-4) & $11(28.21)$ & $8(21.62)$ & \\
\hline Peptic ulcer (Grade 5) & o & $2(5 \cdot 41)$ & $0.2337^{\mathrm{b}}$ \\
\hline Grade o (no visible lesion) & $24(61.54)$ & $22(59 \cdot 45)$ & \\
\hline Grade 1 (mucosal hemorrhages only) & $4(10.26)$ & $5(13 \cdot 51)$ & \\
\hline Grade 2 (one or two erosions) & $7(17 \cdot 95)$ & $3(8.11)$ & \\
\hline Grade 3 (numerous [3-10] numbers of erosions) & $3(7.69)$ & $5(13 \cdot 51)$ & \\
\hline Grade 4 (large [>10] numbers of erosions) & $1(2.56)$ & 0 & \\
\hline Grade 5 (ulcer) & 0 & $2(5.41)$ & \\
\hline $\begin{array}{l}\text { Institute investigator vs. independent evaluator } 1 \\
\text { concordance rate }\end{array}$ & & & 0.4707 \\
\hline $\begin{array}{l}\text { Institute investigator vs. independent evaluator } 2 \\
\text { concordance rate }^{c}\end{array}$ & & & 0.3566 \\
\hline
\end{tabular}

Values are presented as number (\%). Test group, irsogladine maleate (Gaslon $\left.\mathrm{N}^{\circledast} \mathrm{OD}\right), 2 \mathrm{mg}$ twice a day; Control group, placebo drug of irsogladine maleate $2 \mathrm{mg}$ twice a day.

${ }^{\text {a}}$ Fisher's exact test.

${ }^{\mathrm{b}}$ For peptic ulcer, Fisher's exact test.

${ }^{\mathrm{c} C o h e n ' s ~ w e i g h t e d ~} \kappa$ value: not the occurrence, concordance rate of modified Lanza score.

significant difference in incidence of peptic inflammation with MLS of 2 to 4 between the two groups (Table 3). Subgroup analysis did not show any statistically significant difference in incidence of peptic inflammation with MLS of 2 to 4 between groups either.

Incidence or aggravation of peptic ulcer/inflammation by NSAIDs or aspirin treatment at 8 weeks after administering the test drug was assessed by MLS grade change between before and after test drug administration. As a result, changes in the test group were improvement in two (5.13\%), unchanged in 26 (66.67\%), and worsened in 11 (28.21\%) patients. Changes in the control group were improvement in three $(8.11 \%)$, unchanged in $20(54.05 \%)$, and deteriorated in 14 patients $(37.84 \%)$ (Table 4). Therefore, peptic ulcer/inflammation deterioration was prevented slightly by administration of the test drug. However, the difference between the two groups was not statistically significant (unchanged + improvement vs. worsened, $p=0.3717$; unchanged vs. improved vs. worsened, $p=0.5688$ ) (Table 4 ). In subgroup analysis, for all subgroups except $H$. pylori-positive group, administration of test drug showed a tendency to prevent peptic ulcer/inflammation worsening. H. pylori-negative subgroup analysis showed that changes in the test group were improvement in one (3.85\%), unchanged in $19(73.08 \%)$, and deteriorated in six patients (23.08\%). Those in the control group were improvement in three (13.64\%), unchanged in eight (36.36\%), and worsened in 11 patients (50.00\%). Differences between groups were statistically significant (unchanged + improvement vs. worsened, $p=0.0520$; unchanged vs. improvement vs. worsened, $p=0.0247$ ) (Table 4 ).

\section{Secondary efficacy assessment}

(1) Number of acute erosions: The number of acute erosions confirmed by endoscopy at 8 weeks as a secondary efficacy variable was $0.79 \pm 1.82$ in the stomach of the test group, $\mathrm{o}$ in the duodenum of the test group, $1.05 \pm 1.96$ in the stomach of the control group, and $0.14 \pm 0.82$ in the duodenum of the control group. Therefore, a total of $0.79 \pm 1.82$ and $1.19 \pm 2.42$ acute erosions were identified in the test group and control group, respectively. The number of acute erosions tended to be smaller in the test drug group compared to that in the control group. However, the difference between the two was not statistically significant $(p=0.5884$ ) (Supplementary Table 1). In subgroup analysis, for all subgroups except $H$. pylori-positive group, test group showed a tendency to 
Table 4. Changes of modified Lanza score(MLS) at 8 weeks after treatment compared to MLS at baseline

\begin{tabular}{|c|c|c|c|c|}
\hline Variable & Test group & Control group & $p$ value & $p$ value \\
\hline Total & 39 & 37 & $0.3717^{\mathrm{a}}$ & $0.5688^{b}$ \\
\hline Unchanged & $26(66.67)$ & $20(54.05)$ & & \\
\hline Improved & $2(5.13)$ & $3(8.11)$ & & \\
\hline aggravated & $11(28.21)$ & $14(37.84)$ & & \\
\hline Helicobacter pylori + & 13 & 15 & $0.4097^{c}$ & $0.2966^{\mathrm{b}}$ \\
\hline Unchanged & $7(53.85)$ & $12(80.00)$ & & \\
\hline Improved & $1(7.69)$ & $\mathrm{o}$ & & \\
\hline Worsened & $5(38.46)$ & $3(20.00)$ & & \\
\hline Helicobacter pylori- & 26 & 22 & $0.0520^{a}$ & $0.0247^{b}$ \\
\hline Unchanged & $19(73.08)$ & $8(36.36)$ & & \\
\hline Improved & $1(3.85)$ & $3(13.64)$ & & \\
\hline Worsened & $6(23.08)$ & $11(50.00)$ & & \\
\hline
\end{tabular}

Values are presented as number (\%). Test group, irsogladine maleate (Gaslon $\left.\mathrm{N}^{\circledR} \mathrm{OD}\right), 2$ mg twice a day; Control group, placebo drug of irsogladine maleate $2 \mathrm{mg}$ twice a day.

${ }^{a}$ Intergroup comparison (unchanged + improved vs. worsened): chi-square test.

${ }^{b}$ Intergroup comparison (unchanged vs. improved vs. worsened): Fisher's exact test.

${ }^{\mathrm{c}}$ Intergroup comparison (unchanged + improved vs. worsened): Fisher's exact test.

have a smaller number of acute erosions compared to the control group. However, the difference between the two groups was not statistically significant. The number of acute erosions in the stomach and duodenum determined by institutional investigator showed fair ( 0.610 or 0.564 ) and good ( 0.663 or 0.692 ) agreement with the number of acute erosion evaluated by independent evaluator 1 or 2, respectively (Supplementary Table 1).

(2) MDA level changes based on endoscopic biopsy: Changes in MDA levels measured at 8 weeks versus baseline were $-0.22 \pm 0.94 \mathrm{nmol} / \mu \mathrm{L}$ in the test group and $-0.05 \pm 0.70 \mathrm{nmol} / \mu \mathrm{L}$ in the control group. However, the reduction in MDA level was not statistically significant in either group. The reduction in MDA level in the test group at 8 weeks after administration of the test drug was about four times higher than that in the control group. However, the difference between the two was not statistically significant $(p=0.4098)$ (Supplementary Table 2). Similar trends were observed in all subgroups.

(3) Efficacy against edema: After the treatment, changes for edema in the test group were improvement in one (2.56\%), unchanged in 36 (92.31\%), and worsening in two patients $(5.13 \%)$. Changes for edema in the control group were improvement in one $(2.70 \%)$, unchanged in 31 (83.78\%), and deteriorated in five patients (13.51\%).
However, difference was not statistically significant (unchanged + improved vs. worsened, $p=0.2562$; unchanged vs. improved vs. worsened, $p=0.6229$ ) (Table 5). Subgroup analysis showed that administration of test drug tended to prevent edema in all subgroups. In particular, $H$. pylori-negative subgroup analysis showed that 26 patients $(100 \%)$ in the test group were unchanged. However, in the control group, one patient (4.55\%) was improved, 17 (77.27\%) were unchanged, and four (18.18\%) were worsened (unchanged vs. improved vs. worsened, $p=0.0154$; unchanged + improved vs. worsened, $p=0.0376$ ). Their differences were statistically significant (Table 5). Results of edema evaluation by institutional investigators showed fair or poor agreement with results determined by two independent evaluators.

(4) Efficacy against redness: The protection efficacy of test drug against NSAIDs or aspirin-induced redness was evaluated by determining the degree of flares (normal, mild, moderate, severe) through gastroscopy at baseline and the 8th week. As a result, changes in NSAIDs or aspirin-induced redness in the test group were improved in eight (20.51\%), unchanged in 21 (53.85\%), and worsened in 10 patients $(25.64 \%)$. In the control group, those changes were improved in eight (21.62\%), unchanged in 19 (51.35\%), and worsened in 10 patients (27.03\%). There was 
Shim KN, et al. Irsogladine maleate in NSAID-ulcer

Table 5. Efficacy against edema by gastroscopy at 8 weeks after treatment

\begin{tabular}{|c|c|c|c|c|}
\hline Variable & Test group & Control group & $p$ value $^{a}$ & $p$ value $^{b}$ \\
\hline Total & 39 & 37 & 0.2562 & 0.6229 \\
\hline \multicolumn{5}{|l|}{ Baseline } \\
\hline No & $36(92.31)$ & $33(89.19)$ & & \\
\hline Yes & $3(7.69)$ & $4(10.81)$ & & \\
\hline \multicolumn{5}{|l|}{8 Weeks } \\
\hline No & $35(89.74)$ & $29(78.38)$ & & \\
\hline Yes & $4(10.26)$ & $8(21.62)$ & & \\
\hline \multicolumn{5}{|c|}{ Change at 8 weeks from baseline } \\
\hline Unchanged & $36(92.31)$ & $31(83.78)$ & & \\
\hline Improved & $1(2.56)$ & $1(2.70)$ & & \\
\hline Worsened & $2(5.13)$ & $5(13 \cdot 51)$ & & \\
\hline Helicobacter pylori + & 13 & 15 & 0.5833 & 0.3833 \\
\hline \multicolumn{5}{|l|}{ Baseline } \\
\hline No & $11(84.62)$ & $14(93 \cdot 33)$ & & \\
\hline Yes & $2(15.38)$ & $1(6.67)$ & & \\
\hline \multicolumn{5}{|l|}{8 Weeks } \\
\hline No & $10(76.92)$ & $13(86.67)$ & & \\
\hline Yes & $3(23.08)$ & $2(13 \cdot 33)$ & & \\
\hline \multicolumn{5}{|c|}{ Change at 8 weeks from baseline } \\
\hline Unchanged & $10(76.92)$ & $14(93.33)$ & & \\
\hline Improved & $1(7.69)$ & 0 & & \\
\hline Worsened & $2(15.38)$ & $1(6.67)$ & & \\
\hline Helicobacter pylori- & 26 & 22 & 0.0376 & 0.0154 \\
\hline \multicolumn{5}{|l|}{ Baseline } \\
\hline No & $25(96.15)$ & $19(86.36)$ & & \\
\hline Yes & $1(3.85)$ & $3(13.64)$ & & \\
\hline \multicolumn{5}{|l|}{8 Weeks } \\
\hline No & $25(96.15)$ & $16(72.73)$ & & \\
\hline Yes & $1(3.85)$ & $6(27.27)$ & & \\
\hline \multicolumn{5}{|c|}{ Change at 8 weeks from baseline } \\
\hline Unchanged & $26(100)$ & $17(77.27)$ & & \\
\hline Improved & o & $1(4 \cdot 55)$ & & \\
\hline Worsened & O & $4(18.18)$ & & \\
\hline
\end{tabular}

Institute investigator vs. independent evaluator 1 concordance rate

Cohen's weighted $\kappa$ value

$\begin{array}{lr}\text { Baseline } & -0.0585 \\ 8 \text { Weeks } & 0.2549\end{array}$

Institute investigator vs. independent evaluator 2 concordance rate

Cohen's weighted $\kappa$ value

$\begin{array}{lr}\text { Baseline } & -0.0585 \\ 8 \text { Weeks } & 0.1307\end{array}$

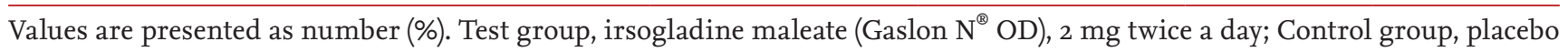
drug of irsogladine maleate $2 \mathrm{mg}$ twice a day.

${ }^{a}$ Intergroup comparison (unchanged + improved vs. worsened): Fisher's exact test.

${ }^{\mathrm{b}}$ Intergroup comparison (unchanged vs. improved vs. worsened): Fisher's exact test. 
no statistically significant difference in redness changes between the two groups (unchanged + improvement vs. worsening, $p=0.8909$; unchanged vs. improved vs. worsening, $p=0.9766$ ). There was no statistically significant difference in subgroup analysis between the two groups either. Results of redness evaluated by institutional investigators showed fair or poor agreement with results determined by two independent evaluators.

(5) Efficacy against haemorrhage: Baseline and 8th week gastroscopies were performed to evaluate the degree of hemorrhage (none, 1 lesion, 2 to 5 lesions, 6 to 10 lesions, or more than 10 lesions). Prevention of NSAIDs or aspirin-induced hemorrhage was evaluated. As a result, changes in hemorrhage in the test group were improved in o (०\%), unchanged in 34 (87.18\%), and aggravated in five patients $(12.82 \%)$. In the control group, these changes were improved in two (5.41\%), unchanged in $31(83.78 \%)$, and aggravated in four patients (10.81\%). There was no statistically significant difference in changes of hemorrhage between the two groups (unchanged + improved vs. aggravated, $p=1.0000$; unchanged vs. improved vs. worsened, $p=0.4361$ ). In subgroup analysis, there was statistically significant difference in hemorrhage changes between the two groups in the $\mathrm{H}$. pylori-positive group (unchanged + improved vs. worsened, $p=0.0349$; unchanged vs. improved vs. worsened, $p=0.0349$ ). However, the number of subjects used in this study was small. In other subgroups, there was no statistical significance in hemorrhage changes between the two treatment groups. Results of hemorrhage evaluation by institutional investigators showed fair or poor agreement with results obtained by two independent evaluators.

(6) Efficacy against subjective symptoms: The frequency and severity of GI symptoms were evaluated using Korean version of the Nepean Dyspeptic Index (NDI). NDI awareness symptom evaluation scale consisted of 10 GI symptoms. The frequency of each question was rated o to 4 points and the severity was scored o to 3 points. The higher the score, the greater the frequency and symptoms. Total score changes of subjective symptom frequency between the 8th week and baseline in test group were improved in $10(25.64 \%)$, unchanged in $13(33.33 \%)$, and deteriorated in 16 patients (41.03\%). In the control group, these changes were improved in 12 (32.43\%), unchanged in 15 (40.54\%), and aggravated in 10 patients $(27.03 \%)$. There was a trend of increased subjective symptom frequency in the test group.
However, the difference between the two groups was not statistically significant (unchanged + improved vs. worsening, $p=0.1985$; unchanged vs. improved vs. worsening, $p=0.4365)$. All subgroups showed similar trends.

As a result of evaluating total score changes for the degree of subjective symptom at the 8th week compared to those at baseline based on case rate, changes in subjective symptoms were improved in eight (20.51\%), unchanged in 16 (41.03\%), and deteriorated in 15 patients (38.46\%) in the test group. In the control group, these changes were improved in 11 (29.73\%), unchanged in 15 (40.54\%), and aggravated in 11 patients (29.73\%). There was no statistically significant different between the two treatment groups (unchanged + improved vs. worsening, $p=0.4226$; unchanged vs. improved vs. worsening, $p=0.5858$ ).

\section{Safety}

A total of 19 adverse events occurred in 16 out of 87 patients in the safety group. There was no statistically significant difference in adverse events between the two treatment groups. Two serious adverse events (10.53\%) occurred. Patients were hospitalized due to chest discomfort and influenza symptoms. However, there was no causal relationship between the two events and the clinical trial drug. GI disorders were the most common (six cases, 6.90\%). At 4 and 8 weeks after administration of the drug for clinical trial, rescue drug was administered $6.55 \pm 11.23$ times in the test group and $6.92 \pm 7.98$ times in the control group at 4 weeks and was administered $5.90 \pm 8.16$ times in the test group and $4.70 \pm 6.94$ times in the control group at 8 weeks. There was no significant difference in the number of rescue drug administration between the two groups.

\section{DISCUSSION}

The present study evaluated the efficacy and safety of irsogladine maleate for preventing NSAIDs or aspirin-induced peptic ulcer and gastritis in patients who were more than 50 years old. There were two cases of peptic ulcer in the placebo group and no peptic ulcer in the test group. However, the difference in the incidence of peptic ulcer between the two groups did not reach statistical significance mainly due to small number of subjects used in this study. Another significant finding was 
that changes in MLS score $(p=0.0247)$ and edema score ( $p=0.0154)$ were statistically significant between before and after treatment in $\mathrm{H}$. pylori-negative patients. This means that the protective effect of irsogladine maleate is prominent when subjects are $H$. pylori-negative. $H$. pylori-infected gastric mucosa or gastric epithelial cell lines have shown inhibition in HKa promotor activity of endogenous or transfected H,K-ATPase [35]. Chronic H. pylori infection in the antrum can up-regulate gastrin and subsequently elevate acid secretion while chronic infection in the corpus can lead to impaired acid secretion by direct suppression of H,K-ATPase or involvement of cytokines such as interleukin-1 $\beta$ or tumor necrosis factor- $\alpha$ [36]. Therefore, direct inhibition of H,K-ATPase, indirect inhibition through cytokines, and loss of parietal cells by ongoing inflammation are three mechanisms associated with low acid secretion in chronic corpus predominant gastritis [37]. In addition, only $40 \%$ of $H$. pylori-negative peptic ulcer Korean patients who received continuous PPI infusion reached target $\mathrm{pH}>6$ for more than $60 \%$ of the time, which was significantly lower than that in the $H$. pylori-positive group at $87.5 \%$ ( $p$ $=0.026)$ [38]. Taken together, these results suggest that adequate acid inhibition might be difficult in $\mathrm{H}$. pylori-negative patients. However, significant protective effect of irsogladine maleate in $\mathrm{H}$. pylori-negative subjects was found in the present study. This suggests that, when NSAIDs or aspirin is unavoidable in patients who are over 50 years old, gastroprotective drug should be added, especially in the absence of $H$. pylori-induced buffer effect. Another supportive evidence for this assumption was that two subjects who developed gastric ulcers while taking NSAIDs or aspirin in the placebo group were $H$. pylori-negative subjects. Our results favour results of previous reports showing that $H$. pylori gastritis is not a risk factor for gastroduodenal damage in subjects taking NSAIDs or aspirin [39-43].

The mechanism of action of irsogladine maleate (Gaslon $\mathrm{N}^{\circledR} \mathrm{OD}$ ) is known to cause mucoprotection through activating intercellular communication and increasing gastric mucosal blood flow, anti-inflammatory action, and maintenance of mucosal hydrophobicity [44-46]. However, primary or secondary outcomes were not significantly different between test group and control groups, although we recruited patients at age of more than 50 years old with an average age of 65.74 years. This might be due to the fact that insufficient number of subjects were recruited to find difference between the two groups. The incidence of peptic ulcer (21.7\%) in the hypothetical placebo group was somewhat high. However, it did not sufficiently reflect the recent tendency of decreasing prevalence of peptic ulcer [47]. Moreover, the protective effect of misoprostol for peptic ulceration was also relatively high in the reference study [24]. In spite of this critical limitation, two cases in the placebo group showed peptic ulcer while none in the test group showed peptic ulcer. These two cases of peptic ulcer were both $H$. pylori-negative patients. In addition, $H$. pylori-negative group showed significant changes in MLS score $(p=0.0247)$ and edema score $(p$ $=0.0154$ ) between before and after the treatment. These results suggest that irsogladine maleate might have potential as a protective agent against NSAIDs or aspirin-induced peptic ulcer and gastritis.

\section{KEY MESSAGE}

1. The widespread use of nonsteroidal anti-inflammatory drugs (NSAIDs) raises the risk of drug side effects, including gastrointestinal damage. The risk of gastric mucosal defects (erosion or petechiae) in patients with long-term use of NSAIDs has been reported to be about $50 \%$.

2. Misoprostol and proton pump inhibitor preparations are usually recommended to prevent NSAID or aspirin-induced peptic ulcer and gastritis, especially for the elderly. However, there are limitations of their usage such as abdominal pain and longterm use related various side effects, respectively.

3. Irsogladine maleate (Gaslon $\mathrm{N}{ }^{\circledR} \mathrm{OD}$, Taejoon Pharm Co. Ltd.) is an enhancer of gastric mucosal protective factors. Our study suggests that its potential as a protective agent against NSAIDs or aspirin-induced peptic ulcer and gastritis in Helicobacter pylori-negative subjects.

\section{Conflict of interest}

This study was funded by a grant from Taejoon Pharm Co. Ltd., Seoul, South Korea. 


\section{REFERENCES}

1. Coxib and traditional NSAID Trialists' (CNT) Collaboration, Bhala N, Emberson J, et al. Vascular and upper gastrointestinal effects of non-steroidal anti-inflammatory drugs: meta-analyses of individual participant data from randomised trials. Lancet 2013;382:769-779.

2. Sostres C, Gargallo CJ, Lanas A. Nonsteroidal anti-inflammatory drugs and upper and lower gastrointestinal mucosal damage. Arthritis Res Ther 2013;15 Suppl 3:S3.

3. Lanza FL. A review of gastric ulcer and gastroduodenal injury in normal volunteers receiving aspirin and other non-steroidal anti-inflammatory drugs. Scand J Gastroenterol Suppl 1989;163:24-31.

4. Kim JJ, Kim N, Lee BH, et al. Risk factors for development and recurrence of peptic ulcer disease. Korean J Gastroenterol 2010;56:220-228.

5. Bae S, Kim N, Kang JM, et al. Incidence and 30-day mortality of peptic ulcer bleeding in Korea. Eur J Gastroenterol Hepatol 2012;24:675-682.

6. Bae S, Shim KN, Kim N, et al. Incidence and short-term mortality from perforated peptic ulcer in Korea: a population-based study. J Epidemiol 2012;22:508-516.

7. Kang JM, Kim N, Kim JH, et al. Effect of aging on gastric mucosal defense mechanisms: ROS, apoptosis, angiogenesis, and sensory neurons. Am J Physiol Gastrointest Liver Physiol 2010;299:G1147-G1153.

8. Sung JJ, Chan FK, Chen M, et al. Asia-Pacific Working Group consensus on non-variceal upper gastrointestinal bleeding. Gut 2011;60:1170-1177.

9. Song HJ, Kwon JW, Kim N, Park YS. Cost effectiveness associated with Helicobacter pylori screening and eradication in patients taking nonsteroidal anti-inflammatory drugs and/or aspirin. Gut Liver 2013;7:182-189.

10. Malfertheiner P, Megraud F, O'Morain CA, et al. Management of Helicobacter pylori infection: the Maastricht IV/ Florence Consensus Report. Gut 2012;61:646-664.

11. Cryer B, Feldman M. Effects of very low dose daily, longterm aspirin therapy on gastric, duodenal, and rectal prostaglandin levels and on mucosal injury in healthy humans. Gastroenterology 1999;117:17-25.

12. Goldstein JL, Lowry SC, Lanza FL, Schwartz HI, Dodge WE. The impact of low-dose aspirin on endoscopic gastric and duodenal ulcer rates in users of a non-selective non-steroidal anti-inflammatory drug or a cyclo-oxygenase-2-selective inhibitor. Aliment Pharmacol Ther
2006;23:1489-1498.

13. Hernandez-Diaz S, Garcia Rodriguez LA. Cardioprotective aspirin users and their excess risk of upper gastrointestinal complications. BMC Med 2006;4:22.

14. Niv Y, Battler A, Abuksis G, Gal E, Sapoznikov B, Vilkin A. Endoscopy in asymptomatic minidose aspirin consumers. Dig Dis Sci 2005;50:78-80.

15. Yeomans ND, Lanas AI, Talley NJ, et al. Prevalence and incidence of gastroduodenal ulcers during treatment with vascular protective doses of aspirin. Aliment Pharmacol Ther 2005;22:795-801.

16. Cheung DY, Jung HY, Song HJ, et al. Guidelines of treatment for non-bleeding peptic ulcer disease. Korean J Gastroenterol 2009;54:285-297.

17. Ali T, Roberts DN, Tierney WM. Long-term safety concerns with proton pump inhibitors. Am J Med 2009;122:896-903.

18. Kyoi T, Noda K, Oka M, Ukai Y. Irsogladine, an anti-ulcer drug, suppresses superoxide production by inhibiting phosphodiesterase type 4 in human neutrophils. Life Sci 2004;76:71-83.

19. Ueda F, Kameda Y, Yamamoto O, Shibata Y. Beta-adrenergic regulation of gap-junctional intercellular communication in cultured rabbit gastric epithelial cells. J Pharmacol Exp Ther 1994;271:397-402.

20. Kyoi T, Oka M, Noda K, Ukai Y. Irsogladine prevents monochloramine-induced gastric mucosal lesions by improving the decrease in mucosal blood flow due to the disturbance of nitric oxide synthesis in rats. J Pharmacol Sci 2003;93:314-320.

21. Sato M, Manabe N, Hata J, et al. Effect of irsogladine maleate on NSAID-induced reduction of gastric mucosal blood flow in anesthetized dogs. Digestion 2009;79:73-78.

22. Tatsumi Y, Tanino M, Kodama T, Kashima K, Katsura M, Okuma S. Irsogladine maleate may preserve gastric mucosal hydrophobicity against ethanol in phospholipids independent way in rats. Jpn J Pharmacol 1998;77:293-299.

23. Desai JC, Sanyal SM, Goo T, et al. Primary prevention of adverse gastroduodenal effects from short-term use of non-steroidal anti-inflammatory drugs by omeprazole 20 mg in healthy subjects: a randomized, double-blind, placebo-controlled study. Dig Dis Sci 2008;53:2059-2065.

24. Graham DY, Agrawal NM, Roth SH. Prevention of NSAID-induced gastric ulcer with misoprostol: multicentre, double-blind, placebo-controlled trial. Lancet 1988;2:1277-1280.

25. Laine L. Nonsteroidal anti-inflammatory drug gastropa- 
thy. Gastrointest Endosc Clin N Am 1996;6:489-504.

26. Laine L. The gastrointestinal effects of nonselective NSAIDs and COX-2-selective inhibitors. Semin Arthritis Rheum 2002;32(3 Suppl 1):25-32.

27. Lee OY, Kang DH, Lee DH, et al. A comparative study of DA-9601 and misoprostol for prevention of NSAID-associated gastroduodenal injury in patients undergoing chronic NSAID treatment. Arch Pharm Res 2014;37:1308-1316.

28. Niwa Y, Nakamura M, Ohmiya N, et al. Efficacy of rebamipide for diclofenac-induced small-intestinal mucosal injuries in healthy subjects: a prospective, randomized, double-blinded, placebo-controlled, cross-over study. J Gastroenterol 2008;43:270-276.

29. Thongbai T. The prevalence of gastroduodenal mucosal injuries in aspirin users. J Med Assoc Thai 2013;96:14231427.

30. Zhang S, Qing Q, Bai Y, et al. Rebamipide helps defend against nonsteroidal anti-inflammatory drugs induced gastroenteropathy: a systematic review and meta-analysis. Dig Dis Sci 2013;58:1991-2000.

31. Jo HJ, Kim N, Nam RH, et al. The effect of Cochinchina momordica seed extract on gastric acid secretion and morphologic change in aged rat stomach. Gut Liver 2013;7:560-568.

32. Lanza FL, Royer GL Jr, Nelson RS, Rack MF, Seckman CC. Ethanol, aspirin, ibuprofen, and the gastroduodenal mucosa: an endoscopic assessment. Am J Gastroenterol 1985;80:767-769.

33. Lanza FL. Endoscopic studies of gastric and duodenal injury after the use of ibuprofen, aspirin, and other nonsteroidal anti-inflammatory agents. Am J Med 1984;77:19-24.

34. Tytgat GN. The Sydney System: endoscopic division. Endoscopic appearances in gastritis/duodenitis. J Gastroenterol Hepatol 1991;6:223-234.

35. Smolka AJ, Backert S. How Helicobacter pylori infection controls gastric acid secretion. J Gastroenterol 2012;47:609618.

36. Beales IL, Calam J. Interleukin 1 beta and tumour necrosis factor alpha inhibit acid secretion in cultured rabbit parietal cells by multiple pathways. Gut 1998;42:227-234.

37. Kim N, Choi YJ. Change of acid secretions, ghrelin and leptin, by H. pylori. In: Kim N, ed. Helicobacter pylori. Singapore: Springer Co., 2016:53-76.

38. Choi KD, Kim N, Jang IJ, et al. Optimal dose of intrave- nous pantoprazole in patients with peptic ulcer bleeding requiring endoscopic hemostasis in Korea. J Gastroenterol Hepatol 2009;24:1617-1624.

39. Goggin PM, Collins DA, Jazrawi RP, et al. Prevalence of Helicobacter pylori infection and its effect on symptoms and non-steroidal anti-inflammatory drug induced gastrointestinal damage in patients with rheumatoid arthritis. Gut 1993;34:1677-1680.

40. Kim JG, Graham DY. Helicobacter pylori infection and development of gastric or duodenal ulcer in arthritic patients receiving chronic NSAID therapy. The Misoprostol Study Group. Am J Gastroenterol 1994;89:203-207.

41. Laine L, Cominelli F, Sloane R, Casini-Raggi V, Marin-Sorensen $M$, Weinstein WM. Interaction of NSAIDs and Helicobacter pylori on gastrointestinal injury and prostaglandin production: a controlled double-blind trial. Aliment Pharmacol Ther 1995;9:127-135.

42. Lanza FL, Evans DG, Graham DY. Effect of Helicobacter pylori infection on the severity of gastroduodenal mucosal injury after the acute administration of naproxen or aspirin to normal volunteers. Am J Gastroenterol 1991;86:735-737.

43. Thillainayagam AV, Tabaqchali S, Warrington SJ, Farthing MJ. Interrelationships between Helicobacter pylori infection, nonsteroidal antiinflammatory drugs and gastroduodenal disease. A prospective study in healthy volunteers. Dig Dis Sci 1994;39:1085-1089.

44. Akagi M, Amagase K, Murakami T, Takeuchi K. Irsogladine: overview of the mechanisms of mucosal protective and healing- promoting actions in the gastrointestinal tract. Curr Pharm Des 2013;19:106-114.

45. Kuramoto T, Umegaki E, Nouda S, et al. Preventive effect of irsogladine or omeprazole on non-steroidal anti-inflammatory drug-induced esophagitis, peptic ulcers, and small intestinal lesions in humans, a prospective randomized controlled study. BMC Gastroenterol 2013;13:85.

46. Murakami K, Okimoto T, Kodama M, et al. Comparison of the efficacy of irsogladine maleate and famotidine for the healing of gastric ulcers after Helicobacter pylori eradication therapy: a randomized, controlled, prospective study. Scand J Gastroenterol 2011;46:287-292.

47. Sung JJ, Kuipers EJ, El-Serag HB. Systematic review: the global incidence and prevalence of peptic ulcer disease. Aliment Pharmacol Ther 2009;29:938-946. 


\section{KJIM}

Supplementary Table 1. Number of acute erosions at 8 weeks after treatment

\begin{tabular}{|c|c|c|c|c|}
\hline Variable & Test group $(\mathrm{n}=39)$ & & Control group $(n=37)$ & $p$ value $^{\mathrm{a}}$ \\
\hline \multicolumn{5}{|l|}{ Stomach } \\
\hline Mean \pm SD & $0.79 \pm 1.82$ & & $1.05 \pm 1.96$ & 0.6021 \\
\hline Median & o & & o & \\
\hline Min-Max & $0-9.00$ & & $0-7.00$ & \\
\hline \multicolumn{5}{|l|}{ Duodenum } \\
\hline Mean \pm SD & 0 & & $0.14 \pm 0.82$ & 0.3171 \\
\hline Median & $\mathrm{O}$ & & o & \\
\hline Min-Max & $0-0$ & & $0-5.00$ & \\
\hline \multicolumn{5}{|c|}{ Stomach + duodenum } \\
\hline Mean \pm SD & $0.79 \pm 1.82$ & & $1.19 \pm 2.42$ & 0.5884 \\
\hline Median & o & & o & \\
\hline Min-Max & $0-9.00$ & & $0-11.00$ & \\
\hline \multicolumn{5}{|c|}{ Institute investigator vs. independent evaluator 1 concordance rate } \\
\hline \multicolumn{5}{|c|}{ Intraclass correlation coefficient } \\
\hline Stomach & & 0.610 & & \\
\hline Duodenum & & 0.663 & & \\
\hline \multicolumn{5}{|c|}{ Institute investigator vs. independent evaluator 2 concordance rate } \\
\hline \multicolumn{5}{|c|}{ Intraclass correlation coefficient } \\
\hline Stomach & & 0.564 & & \\
\hline Duodenum & & 0.692 & & \\
\hline
\end{tabular}

Test group, irsogladine maleate (Gaslon $\left.\mathrm{N}^{\circledast} \mathrm{OD}\right), 2 \mathrm{mg}$ twice a day; Control group, placebo drug of irsogladine maleate 2 mg twice a day. $\mathrm{SD}$, standard deviation.

${ }^{\mathrm{a}}$ Intergroup comparison: Wilcoxon rank sum test. 
Shim KN, et al. Irsogladine maleate in NSAID-ulcer

Supplementary Table 2. Malondialdehyde level changes obtained by endoscopic biopsy at 8 weeks after treatment

\begin{tabular}{|c|c|c|}
\hline Variable & Test group $(n=38)$ & Control group $(n=33)$ \\
\hline \multicolumn{3}{|l|}{ Baseline } \\
\hline Mean \pm SD & $1.23 \pm 1.14$ & $1.23 \pm 0.87$ \\
\hline Median & 0.87 & 0.97 \\
\hline Min-Max & 0.08 to 5.66 & 0.19 to 3.27 \\
\hline \multicolumn{3}{|l|}{8 Weeks } \\
\hline Mean \pm SD & $1.01 \pm 0.94$ & $1.18 \pm 0.75$ \\
\hline Median & 0.65 & 1.21 \\
\hline Min-Max & o to $3 \cdot 55$ & 0.13 to 3.60 \\
\hline \multicolumn{3}{|c|}{ Change at 8 weeks from baseline ${ }^{\mathrm{a}}$} \\
\hline Mean \pm SD & $-0.22 \pm 0.94$ & $-0.05 \pm 0.70$ \\
\hline Median & -0.02 & 0.01 \\
\hline Min-Max & -2.45 to 2.24 & -1.65 to 1.18 \\
\hline$p$ value & $0.2859^{\mathrm{b}}$ & $0.6739^{c}$ \\
\hline$p$ value ${ }^{d}$ & \multicolumn{2}{|c|}{0.4098} \\
\hline
\end{tabular}

Test group, irsogladine maleate (Gaslon $\mathrm{N}^{\circledast} \mathrm{OD}$ ), $2 \mathrm{mg}$ twice a day; Control group, placebo drug of irsogladine maleate $2 \mathrm{mg}$ twice a day.

$\mathrm{SD}$, standard deviation.

${ }^{\mathrm{a}}$ Change $=8$ weeks - baseline.

${ }^{\mathrm{b}}$ Intragroup comparison: Wilcoxon signed rank test.

${ }^{c}$ Intragroup comparison: Paired $t$ test.

${ }^{\mathrm{d}}$ Intergroup comparison: Wilcoxon rank sum test. 